\title{
MEASURING SURGICAL OUTCOMES : NEED OF THE HOUR
}

\section{INTRODUCTION}

Managed health care is taking roots all over the world. Surgical care is now regarded as a commodity which is being increasingly bought by insurance companies for populations. Health plans and payors have begun to focus on the quality of care provided. In an effort to improve patient safety and reduce costs detailed studies by medicare and medicaid services focusing on mortality and morbidity rates are regular features of journals. Surgeons are under increasing pressure to demonstrate patient safety data which meet or even exceed benchmark standards. Surgeons will always be the first to be scrutinized simply because it is much easier to identify mishaps associated with surgery. Records are more detailed and forms are more likely to be filled if it is an operative record. The buyers of health care are getting increasingly aware that variability exists in surgical care quality. Administrators, health care researchers, insurance companies and indeed health professionals are all agreed that our current health care systems are not optimum. They need to be revolutionized. Undoubtedly the safety of our patients must be the pivot of all our endeavours. ${ }^{1}$ The Colorado/Utah study, ${ }^{2}$ Harvard Medical practice study ${ }^{3}$ and the Institute of Medicine report ${ }^{4}$ all document a very high incidence of adverse events in hospitals. Past studies focused on abstracting of charts by personnel remote from the site and after the care was delivered. Ideas are being examined to evaluate the impact of reporting by deputed personnel at the time the service is being delivered. The key questions that need to be answered are:

? How to measure outcomes in surgery?

? Who should do the measuring ?

1. Patients.

2. Buyers of health care.

3. The surgical community itself.

\section{Timilsina D S , Dwaka S*, Majhi P C}

\section{MODELS OF SURGICAL QUALITY CARE IMPROVEMENT PLANS}

We have to single out some areas to focus or else the vast options available will only invite chaos. There are some tools that can be used to measure the quality of surgical care provided. We must learn from the experiences of pioneers in this area of surgery so that we benefit in establishing a quality improvement plan. There are three major quality improvement projects and each has its own system tools that are interesting.

\section{VA NSQIP}

The VA NSQIP (Veteran Affairs, National Surgical Quality Improvement Project) developed by Khuri et $\mathrm{al}^{5}$ over the last 18 years, measured risk-adjusted surgical outcomes of mortality and morbidity for surgical departments through out the VA system. Since its inception mortality and morbidity dropped by $30 \%$ because surgical departments have been provided with feedback that was actionable regarding the results about the quality of care they have been providing. This should be the first prospective study where tracking outcomes lead to improved quality of care. The key feature of the success of the VA NSQIP was the active support of the participating surgeons. This also emphatically proved that surgical errors are generally due to system flaws rather than individual surgeon's level of competence.

\section{Leapfrog Group}

Mortality is the most easily measured and arguably the most basic outcome. It varies significantly as a function of individual surgeon and hospital volume. ${ }^{6,7}$ Recognizing this fact and motivated by the increasing public pressure to demonstrate quality in health care the Leapfrog Group was established in 2000. This group of corporations collectively buy health care

* Manipal Teaching Hospital, Pokhara, Nepal.

Address for correspondence : Dr. Digvijay S. Timilsina 
for 40 million lives in United States. Their aim is to leverage their buying power to stimulate improvements in health care. They buy care from only those systems that implement their evidence based practices linked to higher quality care. Their initial qualifying criteria were ${ }^{8}$

1. Implementation of computerized order entry system for inpatient care.

2. Staffing of ICU by board certified intensivists.

3. Meeting volume based criteria for "Evidence based Hospital Referral for best practices" (EHR), for high risk procedures.

Volume alone as a measure of quality can and has been criticised. ${ }^{9}$ A typical example would be colon resection for cancer. If the presentation was obstruction or it was an elective presentation has a significant bearing on outcome. Patient specific data must be acquired to calculate risk adjusted outcomes to measure surgical quality. Recognizing the evolving science of surgical quality measurements the Leapfrog group revised their criteria for EHR in 2003 to include:

? Minimum volume standards alone for Esophagectomy and Pancreatectomy.

? Volume standards and a process measure (peri-operative beta blockade) for abdominal aortic aneurysm repair.

? Volume standards and risk adjusted mortality rates for Coronary Artery Bypass Graft (CABG) and Percutaneous Cardiac Interventions (PCI).

In 2000, approximately 23,790 patients died in USA undergoing these five procedures. Birkmeyer ${ }^{10}$ reports that full implementation of Leapfrog standards would have averted 7818 of these deaths. CABG (4089). PCI (3016). AAA (356). Esophagectomy (180). Pancreatectomy (177).

This would be $33 \%$ of all the deaths. This represents a three fold increase in number of preventable deaths estimated in a previous report. $^{8}$

There are some very significant conclusions that may be drawn by the Leapfrog system:

1. Applying full criteria, the benefits will be greatest in terms of absolute lives saved for most common procedures (CABG and PCI). Relatively rare complex procedures like Esophagectomy and Pancreatectomy will see a smaller absolute decrease in the number of lives saved. The greatest margin of survival will be between the hospitals that met the criteria and those that did not.

2. We must always remember the potential errors in solely relying on administrative data ${ }^{11}$.

3. Nationwide Inpatient Sample (NIS) relies on hospitals that meet Leapfrog criteria. This will represent a stratified sample of US hospital discharges. Data from 20\% patients will be extrapolated to the rest of the $80 \%$ population. It would be more preferable to compare outcomes between hospitals that meet the criteria and those that do not.

4. Hospital death as an end point will under represent actual death. A high volume tertiary hospital will be more rapidly discharging post operative patients to relatively lower acuity hospital. Thus it may under represent the endpoint itself. Death which in itself is relatively rare event may not be a useful marker for overall quality of care.

5. Perioperative beta-blockade for patients with high cardiac risk may have a risk-reduction up to $50 \%$. The actual value in all patients undergoing AAA repairs can be debated. The hospitals meeting full Leapfrog criteria will actually be effectively using beta-blockade and also obtain 50\% reduction in relative risk is a very big assumption, which is not based on calculable data.

\section{QSS (Quality Surgical Solutions)}

This is a group of surgeons, hospitals and insurance firms. The blueprint for this partnership began with the hand picking of a core of surgeons known for their high-quality and ethical work, low complication rate, cost-effectiveness, and finally, their willingness to undertake such an imposing project. Interested sponsors were Norton Healthcare, Louisville and Anthem Blue Cross Blue Shield. Fifteen common, planned surgical procedures representing over $100 \mathrm{CPT}$ codes were selected and committees, headed by practicing surgeons within the state organization, now named Quality Surgical Solutions (QSS), were formed. A "Best Practice" was determined for each of the procedures, with adherence to these best practices a mechanism for improving quality and controlling cost. Surgeons were educated on each of the best practices and refined them further. The program was initiated July 1, 1998. To monitor adherence to best practices and obtain cost data, the QSS surgeons filled out a report sheet to correspond with each case performed. Since some of the inquiries on the report sheet included items such as back to work date and home health days utilized, the surgeons were asked to report the cases after seeing the patients back in follow-up. In the first year of our program, QSS surgeons performed over 1,300 cases in 15 different categories. The categories are: 1) Partial colon resection. 2) Diabetic foot debridement. 3) Diagnostic and therapeutic endoscopic retrograde cholangiopancreatography (ERCP). 4) Carotid endarterectomy. 5) Laparoscopic cholecystectomy. 6) Transurethral resection prostatectomy (TURP). 7) Extracorporal shock wave lithotripsy (ESWL). 8) Upper and lower endoscopy. 9) Tympanostomy. 10) Tonsillectomy. 11) Inguinal hernia repair. 12) Biopsy of suspected skin cancer. 14) Wide local excision of skin cancer. There is a mechanism in place, based on insurance and hospital 
data, to verify reporting of data by the surgeons. The reporting rate was good, with over $90 \%$ of cases performed by the surgeons accurately reported and entered into the database for analysis. The preliminary results of this endeavor are most encouraging. The complication rate was $1.4 \%$ overall. Some specific examples of quality and cost containment are worth special mention..$^{12}$ Of the 290 colonoscopies performed, 184 were found to be abnormal and 5 new cancers were found during the year. For 22 ESWL's a total of only 3 hospital days were used. During the study period, 105 laparoscopic cholecystectomies were performed. The average length of stay was 0.57 days. No common bile duct injuries occurred. Of the 85 therapeutic ERCPs, all were considered successful and only 2 cases of pancreatitis occurred. There were no myocardial infarctions or cerebrovascular accidents in the peri-operative period of the 15 carotid endarecterectomies. Of the 77 patients undergoing either an excision of a known skin cancer or biopsy of a suspected skin cancer, $96 \%$ received counseling regarding skin cancer prevention in the form of a lecture from the surgeon. The early cost data is equally impressive. Based on data from the Norton Hospital, in the first quarter of 1998, QSS surgeons were considerably more cost-effective than other surgeons in their performance of over 250 cases during that time span. In fact, if all of the procedures done in the last quarter of 1998 were performed by QSS surgeons, the hospital would have saved over $\$ 250,000 .{ }^{12}$ Given the high quality and initial cost savings enjoyed by this joint venture of surgeons, QSS is actively expanding. Ten new best practices have been added and additional surgeons incorporated included specialists such as orthopedic surgeons and hand surgeons. The new cases now include emergency operations (appendectomy) and some non-operative management data, such as medical and behavioral management of carpal tunnel syndrome.

Given the continuing economic pressure imposed on health care providers, improvement of quality with concurrent cost containment is paramount and additional efforts will be put on this project. The excellent model put by Shively et $\mathrm{a}^{13}$ is a very good effort. They defined quality as the best surgical outcome as a function of comparable cost. They have developed a simplified protocol and report form that focused on factors of care that impacted quality system to track outcomes and put up recommendations that can be used by all for starters. They have focused on a wide range of targets that must be strived for:

? Improved classification of indications for systemic prophylactic antibiotic and reduction in the variety of drugs used.

? Shortened length of stay for standard procedures.

? Adherence to strict indications for selective operative procedures.

? Less use of costly diagnostic procedures.

? Decreased use of disposable laparoscopic devices.

? Decreasing use of expensive drugs.

? Develop a method of comparing one-surgeon hospital with his peers.

The purchasers of surgical care are closely watching us. We may not endorse their findings but our clients are using these data in making purchasing decisions. The science of measuring surgical outcomes is improving all along. The NSQIP methodology is being tested in a three year trial funded by AHRQ (Agency of Health Research and Quality) and sponsored by (ACS) American College of Surgeons. It's anticipated that NSQIP will be made available to surgical departments all over as part of ACS program. Even though this system cannot evaluate an individual surgeon's performance, which is its final goal.

\section{CONCLUSION}

As physicians target blood pressure to reduce myocardial infarctions, surgeons need to target errors to improve quality. It is very important that we track our own outcomes and make necessary changes to bring about quality improvements before someone else tells us to.

\section{REFERENCES}

1 Weingart, S.N., Lezzoni, L.I.: Looking for medical injuries: while the light is bright. JAMA 2003; 290: 1917-1919.

2. Gawande $\mathrm{AA}$, Thomas $\mathrm{EJ}$, Zinner $\mathrm{MJ}$, et al. The incidence and nature of surgical adverse events in Colorado and Utah in 1992. Surgery. 1999; 126: 66-75.

3. Brennan $\mathrm{TA}$, Leape $\mathrm{LL}$, Laird MM, et al. Incidence of adverse events and negligence in hospitalized patients: results of the Harvard Medical Practice Study I. N Engl J Med. 1991; 324: 370-376.

4. Kohn LT, Corrigan J, Donaldson MS. To Err Is Human: Building a Safer Health System. Washington, DC: National Academy Press, 2000.

5. Khuri, S.F., Daley, J., Henderson, W.G.: The comparative assessment and improvement in quality surgical care in the Department of Veteran's Affairs. Arch Surg 2002; 137: 2027.

6. Birkmeyer, J.D., Siewers, A.E., Finlayson, E.V., Stukel, T.A., Lucas, F.L., Batista, I., et al.: Hospital volume and surgical mortality in United States. N Engl J Med 2002; 346: 1128-1137.

7. Birkmeyer, J.D., Stukel, T.A., Siewers, A.E., Goodney, P.P., and Lucas, F.L.: Surgeon volume and operative mortality in United States. N Engl J Med 2003; 349: 2117-2127. 
8. Birkmeyer, J.D., Finlayson, E.V., Birkmeyer, C.M. : Volume based standards for high risk surgical procedures: Potential benefits of Leapfrog initiative. Arch Surg 2002; 137: 20-27.

9. Christian, C.K., Gustafson, M.L., Betensky, R.A., Daley, J., and Zinner, M.J.: The Leapfrog volume criteria may fall short in identifying high quality surgical centers. Ann Surg; 2003: 238: $447-455$.

10. Birkmeyer, J.D., and Dinnick, B.J.: Potential benefits of the new Leapfrog standards: effect of process an outcome measures. Surgery 2004; 135 (6) : 569-575.
11. Hunt, J.P., Chen, G.S., Hunter, C., Wright, M.J., Wang, Y.Z., Steeb, G., et al.: Accuracy of administrative data in trauma: splenic injuries as an example. J Trauma 2000; 49: 679-689.

12. http://www.qualitysurgical.com

13. Shively, E.H., Heine, J.M., Schell, R.H., Shanpe, N., Garrison, N., Kenneth, J.S., Polk, $\mathrm{D}$ C., et al.: Practicing surgeons lead in quality care, safety, and cost control. Ann Surg 2004; 239 (6) : $752-762$.

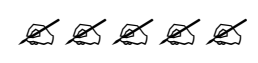

\section{Eastern Regional Ophthalmic Conference}

\section{Theme: Be a good user and producer of research}

It is our pleasure to announce that the III Eastern Regional Ophthalmic Conference is going to be held at Mechi Eye Care Center, Birtamod, Jhapa on April 23-24, 2005. The first and the second ones were held at the Sagarmatha Chaudhary Eye Hospital, Lahan and B P Koirala Instuitute of Health Sciences, Dharan, Sunsari, Nepal respectively.

\section{Important points:}

Venue: Mechi Eye Care Center, Birtamod, Jhapa

Dates: $24^{\text {th }}-25^{\text {th }}$ April 2005.

\section{Last Date for abstract submission}

by e-mail: mecc@ntc.net.np or

by fax: 0023-541492

: $30^{\text {th }}$ March 2005.

Last date for free registration : March 25, 2005. Free registration includes lunch and dinner.

Please confirm your registration by

fax (00977-23-541492),

phone (00977-23-540153) or

E-mail (mecc@ntc.net.np) latest by March 25, 2005.

Accommodation booking facility available on request.

Guest faculties from Nepal and India.

\section{Corresponding address}

\section{Dr. Daki Sherpa, MD}

\section{Organizing secretary}

III Eastern Regional Ophthalmic Conference

Mechi Eye Care Center, Birtamod, Jhapa

E-mail: mecc@ ntc.net.np; Fax: 00977-23-541492, phone: 00977-23-540153 